\title{
On periodic Stokesian Hele-Shaw flows with surface tension
}

\author{
J. ESCHER and B.-V. MATIOC \\ Institute of Applied Mathematics, Leibniz University of Hanover, Welfengarten 1, \\ D-30167 Hanover, Germany \\ email: escher@ifam.uni-hannover.de; matioc@ifam.uni-hannover.de
}

(Received 27 November 2007; revised 7 July 2008; first published online 10 September 2008)

\begin{abstract}
In this paper we consider a $2 \pi$-periodic and two-dimensional Hele-Shaw flow describing the motion of a viscous, incompressible fluid. The free surface is moving under the influence of surface tension and gravity. The motion of the fluid is modelled using a modified version of Darcy's law for Stokesian fluids. The bottom of the cell is assumed to be impermeable. We prove the existence of a unique classical solution for a domain which is a small perturbation of a cylinder. Moreover, we identify the equilibria of the flow and study their stability.
\end{abstract}

\section{Introduction}

In a Stokesian fluid the stress tensor is a continuous function of the deformation tensor and the viscosity depends in general non-linearly on the gradient of the velocity field. The Newtonian fluid is a linear Stokesian fluid for which the viscosity is constant. NonNewtonian fluids play an important role in industrial processes since many polymer solutions and molten polymers are fluids of that type.

Despite their importance in applications, the mathematical understanding of the full problem is far from being complete. For this reason, we study a rather simple situation: fluid flows in a two-dimensional setting of a vertical Hele-Shaw cell. The model is based on a non-Newtonian version of Darcy's law which has been derived in ref. [14]. The liquid phase and the gas phase are separated by a sharp interface. Our main concern is the determination of the motion of this interface, i.e. we have to solve a moving boundary problem.

Similar models are used to describe flows in porous media in refs. [6-9], particularly the dynamic of incompressible fluids in deformable porous media (see ref. [3]). Our paper is a continuation of these studies. In addition to the common Newtonian liquids, the analysis presented in this paper allows us to consider a large class of Stokesian fluids in this model, e.g. Oldroyd-B fluids and power law fluids. In comparison to the Newtonian case the mathematical problem becomes more involved because the generalized Darcy law leads to a nonlinear elliptic boundary value problem for the velocity potential, whereas for Newtonian fluids this potential is harmonic. 


\subsection{The mathematical model}

Let $\alpha \in(0,1)$ be fixed. We define the set of admissible functions

$$
\mathscr{U}:=\left\{f \in C^{4+\alpha}\left(\mathbb{S}^{1}\right): \min _{x \in \mathbb{S}^{1}} f(x)>0\right\},
$$

and denote by $h^{k+\alpha}\left(\mathbb{S}^{1}\right)$ the closure of $C^{\infty}\left(\mathbb{S}^{1}\right)$ in $C^{k+\alpha}\left(\mathbb{S}^{1}\right), k \in \mathbb{N}$. Here, $\mathbb{S}^{1}$ is the unit circle. The fluid domain $\Omega_{f}$ is defined for $f \in \mathscr{U}$ as the set

$$
\Omega_{f}:=\left\{(x, y): x \in \mathbb{S}^{1}, 0<y<f(x)\right\},
$$

and the boundary components of $\Omega_{f}$ are

$$
\Gamma_{f}:=\left\{(x, f(x)): x \in \mathbb{S}^{1}\right\}, \quad \Gamma_{0}:=\mathbb{S}^{1} \times\{0\} .
$$

Further, we denote by $v$ the outward normal of $\partial \Omega_{f}$. For simplicity we identify functions on $\mathbb{S}^{1}$ with $2 \pi$-periodic functions in $\mathbb{R}$. The fluid is modelled using a modified Darcy law

$$
v=-\frac{D u}{\bar{\mu}\left(|D u|^{2}\right)},
$$

cf. ref. [11], where

$$
u(x, y)=\frac{p(x, y)}{g \cdot \rho}+y, \quad(x, y) \in \Omega_{f}
$$

is the so-called velocity potential, $v$ is the velocity field, $p$ is the pressure distribution within the fluid's body, $D u=\left(u_{1}, u_{2}\right)$ is the gradient of $u, g$ is the gravitational constant and $\rho$ is the density of the fluid.

Denoting by $\mu \in C^{\infty}([0, \infty),(0, \infty))$ the viscosity of the fluid, we assume that the function $[0, \infty) \ni r \mapsto h(r):=r \mu^{2}(r)$ is invertible, that is $\mu(r)+2 r \mu^{\prime}(r)>0$ for $r \geqslant 0$. The effective viscosity $\bar{\mu}$, see ref. [11], is defined by

$$
\frac{1}{\bar{\mu}(r)}:=c_{\mu} \int_{-1}^{1} \frac{s^{2}}{\widetilde{\mu}\left(r s^{2}\right)} d s,
$$

where $c_{\mu}$ is a positive constant and $\widetilde{\mu}:=\mu \circ h^{-1}$. The fluid is assumed to be incompressible $(\operatorname{div} v=0)$, thus we get

$$
\operatorname{div}\left(\frac{D u}{\bar{\mu}\left(|D u|^{2}\right)}\right)=0 \quad \text { in } \quad \Omega_{f} .
$$

The free surface $\Gamma_{f}$ separating the fluid from air, at pressure normalized to zero, moves under the influence of surface tension and gravity. At each point on the interface $\Gamma_{f}$ the pressure has a jump according to the formula

$$
p_{\text {int }}-p_{\text {out }}=-\sigma \kappa_{f}
$$

where $p_{\text {out }}$ is the pressure outside the fluid, $p_{\text {int }}$ the pressure inside the fluid domain $\Omega_{f}, \kappa_{f}$ the curvature of $\Gamma_{f}$ and $\sigma$ the surface tension coefficient (see ref. [14], (2.38b)). Denoting 
by $\gamma$ the ratio of $\sigma$ and $g \rho$ we obtain

$$
u=\frac{p}{g \rho}+f=-\frac{\sigma}{g \rho} \kappa_{f}+f=-\gamma \kappa_{f}+f \quad \text { on } \quad \Gamma_{f} .
$$

The bottom of the Hele-Shaw cell is impermeable, thus we have no flux on the fixed boundary component $\Gamma_{0}$,

$$
\partial_{v} u=0 \quad \text { on } \Gamma_{0} .
$$

In order to derive an evolution equation for the free surface we assume that a particle located on the interface $\Gamma_{f}$ remains on the interface as time progresses. The free surface $\Gamma_{f}$ is implicitly given by $F(t, z)=0$, where $z=(x, y)$ and $F(t, z)=y-f(t, x)$. Taking the derivative of this equation with respect to the time variable $t$ and using once again the generalized Darcy's law, we obtain

$$
\partial_{t} f+\frac{\sqrt{1+\partial_{x} f^{2}}}{\bar{\mu}\left(|D u|^{2}\right)} \partial_{v} u=0 \quad \text { on } \quad \Gamma_{f} .
$$

Summarizing, we arrive at the following moving boundary problem

$$
\begin{aligned}
\operatorname{div}\left(\frac{D u}{\bar{\mu}\left(|D u|^{2}\right)}\right) & =0 & & \text { in } \quad \Omega_{f(t)}, \quad t \geqslant 0, \\
\partial_{v} u & =0 & & \text { on } \quad \Gamma_{0}, \quad t \geqslant 0, \\
u & =-\gamma \kappa_{f}+f & & \text { on } \quad \Gamma_{f(t),} t \geqslant 0,
\end{aligned}
$$

where $f_{0}$ is the initial data.

We set $\mathscr{V}:=\mathscr{U} \cap h^{4+\alpha}\left(\mathbb{S}^{1}\right)$. A pair $(u, f)$ is called a classical Hölder solution of (1) on $[0, T]$, with $T>0$, if

$$
\begin{aligned}
& f \in C([0, T], \mathscr{V}) \cap C^{1}\left([0, T], h^{1+\alpha}\left(\mathbb{S}^{1}\right)\right), \\
& u(\cdot, t) \in \operatorname{buc}^{2+\alpha}\left(\Omega_{f(t)}\right), t \in[0, T]
\end{aligned}
$$

and if $(u, f)$ satisfies the equations (1) pointwise. For $f \in \mathscr{U}$, the space ${ }^{1} \operatorname{buc}^{2+\alpha}\left(\Omega_{f}\right)$ is defined as the closure of $\operatorname{BUC}^{\infty}\left(\Omega_{f}\right)$ in $\operatorname{BUC}^{2+\alpha}\left(\Omega_{f}\right)$.

We denote by $\boldsymbol{Q}$ the quasilinear operator

$$
Q u:=\operatorname{div}\left(\frac{D u}{\bar{\mu}\left(|D u|^{2}\right)}\right),
$$

acting on twice-differentiable functions on deformed cylinders $\Omega_{f}$, or $G_{f}$, where $f \in \mathscr{U}$ and

$$
G_{f}:=\left\{(x, y): x \in \mathbb{S}^{1},-f(x)<y<f(x)\right\}
$$

1 The notation buc or BUC stands for 'bounded and uniformly continuous'. 
The coefficients of the quasilinear operator $u \longmapsto Q u=a_{i j}(D u) u_{i j}$ are

$$
a_{i j}\left(x_{1}, x_{2}\right)=\frac{\delta_{i j}}{\bar{\mu}\left(x_{1}^{2}+x_{2}^{2}\right)}-\frac{2 x_{i} x_{j} \bar{\mu}^{\prime}\left(x_{1}^{2}+x_{2}^{2}\right)}{\bar{\mu}^{2}\left(x_{1}^{2}+x_{2}^{2}\right)}, \quad 1 \leqslant i, j \leqslant 2,\left(x_{1}, x_{2}\right) \in \mathbb{R}^{2} .
$$

The eigenvalues of the matrix $\left[a_{i j}(x)\right]_{1 \leqslant i, j \leqslant 2}, x \in \mathbb{R}^{2}$ are

$$
\lambda_{1}(x)=\frac{1}{\bar{\mu}\left(|x|^{2}\right)}, \quad \lambda_{2}(x)=\frac{1}{\bar{\mu}\left(|x|^{2}\right)}-\frac{2|x|^{2} \bar{\mu}^{\prime}\left(|x|^{2}\right)}{\bar{\mu}^{2}\left(|x|^{2}\right)},
$$

and we have

$$
c|\xi|^{2} \leqslant a_{i j}(x) \xi_{i} \xi_{j} \leqslant C|\xi|^{2}, \forall \xi=\left(\xi_{1}, \xi_{2}\right) \in \mathbb{R}^{2}, x \in \mathbb{R}^{2},
$$

if we assume that there exist positive constants $c$ and $C$ such that

$$
\begin{aligned}
& \left(A_{1}\right) c \leqslant \frac{1}{\bar{\mu}(r)} \leqslant C \quad \forall r \geqslant 0, \\
& \left(A_{2}\right) c \leqslant \frac{1}{\bar{\mu}(r)}-\frac{2 r \bar{\mu}^{\prime}(r)}{\bar{\mu}^{2}(r)} \leqslant C, \quad \forall r \geqslant 0 .
\end{aligned}
$$

It can be shown, cf. ref. [4], that $\left(A_{1}\right)$ and $\left(A_{2}\right)$ hold, provided there exist positive constants $m_{\mu}$ and $M_{\mu}$ such that

$$
\begin{aligned}
& \left(V_{1}\right) m_{\mu} \leqslant \mu(r) \leqslant M_{\mu}, \\
& \left(V_{2}\right) m_{\mu} \leqslant \mu(r)+2 r \mu^{\prime}(r) \leqslant M_{\mu},
\end{aligned}
$$

for all $r \geqslant 0$. If the viscosity $\mu$ is constant the fluid is called Newtonian. The class of Stokesian, sometimes also called non-Newtonian, fluids satisfying $\left(V_{1}\right)$ and $\left(V_{2}\right)$ is quite large. This class includes particularly numerous Oldroyd-B and power law fluids. For Oldroyd-B fluids with viscosity given by

$$
\mu(r)=v_{\infty}+\left(v_{0}-v_{\infty}\right) \frac{1+\ln (1+\lambda r)}{1+\lambda r}, \quad r \geqslant 0,
$$

where $\lambda>0$ is a material constant and $v_{0}>v_{\infty}>0$, relations $\left(V_{1}\right)$ and $\left(V_{2}\right)$ hold if $\left(e^{2}+1\right) v_{\infty}>v_{0}$. The power law fluids with viscosity laws:

$$
\mu(r)=v_{\infty}+v_{0}\left(1+r^{2}\right)^{s / 4}, \quad \text { or } \quad \mu(r)=v_{\infty}+v_{0}(1+r)^{s / 2}, \quad r \geqslant 0,
$$

where $v_{0}$ and $v_{\infty}$ are positive and $s \leqslant 0$, belong to this class if $s \in[-1,0]$. The examples mentioned earlier are all shear thinning fluids, i.e. the viscosity decreases with the shear rate. The shear thickening fluid with viscosity

$$
\mu(r)=\mu_{0} \frac{\beta r+r_{0}}{r+r_{0}}, \quad r \geqslant 0,
$$

where $r_{0}>0, \beta \geqslant 1$ and $\mu_{0}>0$, satisfies $\left(V_{1}\right)$ and $\left(V_{2}\right)$ for any choice of the parameters $r_{0}, \mu_{0}$ and $\beta$. 
Thus, if $\left(V_{1}\right)$ and $\left(V_{2}\right)$ hold, then $\boldsymbol{Q}$ is a uniformly elliptic quasilinear operator in $\mathbb{R}^{2}$. Let us now consider the moving boundary problem

$$
\begin{aligned}
& \begin{aligned}
\operatorname{div}\left(\frac{D u}{\bar{\mu}\left(|D u|^{2}\right)}\right) & =0 & & \text { in } \quad G_{f(t)}, \quad t \geqslant 0, \\
u & =-\gamma \kappa_{f}+f & & \text { on } \partial G_{f(t)}, \quad t \geqslant 0,
\end{aligned} \\
& \partial_{t} f(t, \cdot)+\frac{\sqrt{1+\partial_{x} f^{2}(t, \cdot)}}{\bar{\mu}\left(|D u(\cdot, f(t, \cdot))|^{2}\right)} \partial_{v} u(\cdot, f(t, \cdot))=0 \quad \text { on } \mathbb{S}^{1}, \quad t>0,
\end{aligned}
$$

A pair $(u, f)$ is called a classical Hölder solution of $(2)$ on $[0, T]$, with $T>0$, if

$$
\begin{aligned}
& f \in C([0, T], \mathscr{V}) \cap C^{1}\left([0, T], h^{1+\alpha}\left(\mathbb{S}^{1}\right)\right), \\
& u(\cdot, t) \in \operatorname{buc}^{2+\alpha}\left(G_{f(t)}\right), t \in[0, T],
\end{aligned}
$$

and if $(u, f)$ satisfies the equations (2) pointwise. Given $f \in \mathscr{V}$, buc ${ }^{2+\alpha}\left(G_{f}\right)$ stands again for the closure of $\operatorname{BUC}^{\infty}\left(G_{f}\right)$ in $\operatorname{BUC}^{2+\alpha}\left(G_{f}\right)$.

Let $f \in \mathscr{V}$ be given and denote by $u \in \operatorname{buc}^{2+\alpha}\left(G_{f}\right)$ the unique solution of the quasilinear Dirichlet problem

$$
\begin{aligned}
\boldsymbol{Q u} & =0 & \text { in } & G_{f}, \\
u & =-\gamma \kappa_{f}+f & \text { on } & \partial G_{f} .
\end{aligned}
$$

Because of the symmetry of the domain $G_{f}$ and of the boundary conditions we obtain $u(x,-y)=u(x, y)$ for all $(x, y) \in G_{f}$, thus the restriction of $u$ to $\Omega_{f}$ is the unique solution of the mixed boundary problem

$$
\begin{aligned}
& \boldsymbol{Q} u=0 \quad \text { in } \Omega_{f}, \\
& \partial_{v} u=0 \quad \text { on } \Gamma_{0} \text {, } \\
& u=-\gamma \kappa_{f}+f \quad \text { on } \quad \Gamma_{f} .
\end{aligned}
$$

We deduce that there exists a one-to-one correspondence between solutions to (1) and (2) Namely, if $(u, f)$ is a solution to (1) on the interval $[0, T], T>0$, then for each $t \in[0, T]$ the velocity potential $u(\cdot, t) \in \operatorname{buc}^{2+\alpha}\left(\Omega_{f(t)}\right)$ can be extended by reflection on the entire domain $G_{f(t)}$. We obtain in this manner a solution to (2). Moreover, if $(\widetilde{u}, f)$ is a solution to (2) on the interval $[0, T], T>0$, we can restrict for $t \in[0, T]$ the function $\widetilde{u}(\cdot, t)$ to $\Omega_{f(t)}$, and thus obtain a solution to (1). It is more convenient to treat (2), because we have in this case a Dirichlet problem for the velocity potential.

The uniqueness of the solutions to (3) and (4) can be shown using the same method as in the proof of Theorem 9.2 in ref. [10]. The existence of the solutions will be discussed later.

The main results of this paper are as follows.

Theorem 1.1 (Existence and uniqueness) Assume that $\left(A_{1}\right)$ and $\left(A_{2}\right)$ hold.

(a) Let $c$ be a positive constant. Then we find an open neighbourhood $\mathcal{O}$ of $c$ in $\mathscr{V}$ such that, for each $f_{0} \in \mathcal{O}$, problem (2) has a classical Hölder solution $(u, f)$ on an interval $[0, T]$ with $T>0$. Moreover, there exists a constant $\delta \in(0,1)$ such that $f \in C_{\delta}^{\delta}\left((0, T], h^{4+\alpha}\left(\mathbb{S}^{1}\right)\right)$. 
(b) Let $\left(u_{1}, f_{1}\right)$ and $\left(u_{2}, f_{2}\right)$ be solutions of (2) with $f_{1} \in C_{\delta_{1}}^{\delta_{1}}\left((0, T], h^{4+\alpha}\left(\mathbb{S}^{1}\right)\right), \delta_{1} \in(0,1)$ and $\left.f_{2} \in C_{\delta_{2}}^{\delta_{2}}\left((0, T], h^{4+\alpha}\left(\mathbb{S}^{1}\right)\right)\right), \delta_{2} \in(0,1)$. If $f_{1}([0, T]) \subset \mathcal{O}$ and $f_{2}([0, T]) \subset \mathcal{O}$, then $\left(u_{1}, f_{1}\right)=\left(u_{2}, f_{2}\right)$.

For the definition of the weighted Hölder spaces $C_{\gamma}^{\gamma}\left((0, T], h^{4+\alpha}\left(\mathbb{S}^{1}\right)\right), \gamma \in(0,1)$, see, e.g., ref. [12].

Theorem 1.1 guarantees the existence and uniqueness of local solutions, provided they are sufficiently near to a cylinder initially. In Section 4 first we will prove a conservation law for the fluid volume. We further determine the steady-state solutions of the system (1) and prove that the equilibrium $(c, c)$, where $c \in \mathbb{R}_{>0}$, attracts exponentially solutions of (1) which correspond to initial data $f_{0} \in c+\widetilde{h}^{4+\alpha}\left(\mathbb{S}^{1}\right)$ sufficiently closed to $c$. Here $\widetilde{h}^{4+\alpha}\left(\mathbb{S}^{1}\right)=\left\{f \in h^{4+\alpha}\left(\mathbb{S}^{1}\right): \int_{\mathbb{S}^{1}} f d x=0\right\}$. Having $f_{0} \in c+\widetilde{h}^{4+\alpha}\left(\mathbb{S}^{1}\right)$ means the domains $\Omega_{f_{0}}$ and $\Omega_{c}$ contain the same amount of liquid. More precisely we state:

Theorem 1.2 (Exponential stability) For any $\omega<(1+\gamma) \tanh c / \bar{\mu}(0)$, there are positive constants $r$ and $M$ such that for any $f_{0} \in c+\widetilde{h}^{4+\alpha}\left(\mathbb{S}^{1}\right)$ with $\left\|f_{0}-c\right\|_{C^{4+\alpha}\left(\mathbb{S}^{1}\right)} \leqslant r$ solution to (1) exists and the estimate

$$
\|f(t)-c\|_{C^{4+\alpha}\left(\mathbb{S}^{1}\right)}+\left\|f^{\prime}(t)\right\|_{C^{1+\alpha}\left(\mathbb{S}^{1}\right)} \leqslant M e^{-\omega t}\left\|f_{0}-c\right\|_{C^{4+\alpha}\left(\mathbb{S}^{1}\right)}, \quad \forall t \geqslant 0
$$

holds. Moreover, the volume of the domain saturated by fluid is constant in time,

$$
\int_{\mathbb{S}^{1}} f(t) d x=c, \quad \forall t \geqslant 0 .
$$

The new feature of the present paper compared to the problem studied in ref. [4] is the inclusion of surface tension effects. From the analytical point of view this implies that the full problem (1) is governed by a nonlinear evolution equation of third order, whereas the problem studied in ref. [4] is of first order.

Furthermore, our analysis shows the regularizing effect of surface tension since the value $(1+\gamma) \tanh c / \bar{\mu}(0)$ from Theorem 1.2 tends to infinity as $\gamma$ increases to infinity. Such an effect cannot be observed for the system studied in ref. [4].

\subsection{The transformed problem}

In order to solve problem (2), first we have to study the dependence of the solution $u$ to (3) on the variable $f \in \mathscr{V}$. This is quite a difficult task because $u$ is defined on the domain $G_{f}$, and the domain depends on $f$. Therefore, we transform the problem on a fixed domain $G:=\mathbb{S}^{1} \times(0,2)$. For $f \in \mathscr{U}$, let $\phi_{f} \in \operatorname{BUC}^{4+\alpha}\left(G, G_{f}\right)$ be defined by

$$
\phi_{f}(x, y):=(x,(1-y) f(x)),(x, y) \in G
$$


It is clear that $\phi_{f}$ is a global diffeomorphism mapping $G$ onto $G_{f}$. We introduce the push-forward and pull-back operators induced by $\phi_{f}$ :

$$
\begin{aligned}
& \phi_{f}^{*}: \operatorname{BUC}\left(G_{f}\right) \rightarrow \operatorname{BUC}(G), u \longmapsto u \circ \phi_{f}, \\
& \phi_{*}^{f}: \operatorname{BUC}(G) \rightarrow \operatorname{BUC}\left(G_{f}\right), v \longmapsto v \circ \phi_{f}^{-1},
\end{aligned}
$$

and the transformed operators $\mathscr{A}(f)$ and $\mathscr{B}$, acting on $\operatorname{BUC}^{2}(G)$, respectively, on $\mathscr{U} \times \mathrm{BUC}^{2+\alpha}(G)$ by

$$
\begin{aligned}
& \mathscr{A}(f):=\phi_{f}^{*} \circ \boldsymbol{Q} \circ \phi_{*}^{f}, \\
& \mathscr{B}(f, v)(x):=\frac{D\left(\phi_{*}^{f} v\right)}{\bar{\mu}\left(\left|D\left(\phi_{*}^{f} v\right)\right|^{2}\right)}(x, f(x)) \cdot n(x), x \in \mathbb{S}^{1},
\end{aligned}
$$

with $n(x):=\left(-f^{\prime}(x), 1\right), x \in \mathbb{S}^{1}$.

We arrive at the problem

$$
\begin{aligned}
& \mathscr{A}(f) v=0 \quad \text { in } G \times[0, \infty), \\
& v=-\gamma \kappa_{f}+f \quad \text { on } \quad \partial G \times[0, \infty), \\
& \partial_{t} f+\mathscr{B}(f, v)=0 \quad \text { on } \mathbb{S}^{1} \times(0, \infty) \text {, } \\
& f(0)=f_{0} .
\end{aligned}
$$

Given $f \in \mathscr{V}$, the operator $\mathscr{A}(f)$ is uniformly elliptic and

$$
\mathscr{A}(f) v=b_{i j}(y, f, D v) v_{i j}+b(y, f, D v) v_{2} \quad \forall v \in \operatorname{BUC}^{2}(G) .
$$

Here, we use the summation convention and $v_{i}$ and $v_{i j}$ stand for partial derivatives of $v$. Furthermore, the coefficients $b_{i j}$ and $b$ are given by

$$
\begin{aligned}
b_{11}(y, f, D v) & =a_{11}\left(D_{f} v\right) \\
b_{12}(y, f, D v) & =b_{21}(y, f, D v)=\frac{(1-y) f^{\prime}}{f} a_{11}\left(D_{f} v\right)-\frac{1}{f} a_{12}\left(D_{f} v\right), \\
b_{22}(y, f, D v) & =\frac{(1-y)^{2} f^{\prime 2}}{f^{2}} a_{11}\left(D_{f} v\right)-\frac{2(1-y) f^{\prime}}{f^{2}} a_{12}\left(D_{f} v\right)+\frac{1}{f^{2}} a_{22}\left(D_{f} v\right), \\
b(y, f, D v) & =(1-y)\left(\frac{f^{\prime \prime}}{f}-\frac{2 f^{\prime 2}}{f^{2}}\right) a_{11}\left(D_{f} v\right)+\frac{2 f^{\prime}}{f^{2}} a_{12}\left(D_{f} v\right),
\end{aligned}
$$

where $D_{f} v:=\left(v_{1}+\frac{(1-y) f^{\prime}}{f} v_{2},-\frac{1}{f} v_{2}\right)$ for $f \in \mathscr{U}, v \in \operatorname{BUC}^{2}(G)$ and $y \in[0,2]$. A pair $(v, f)$ is a classical Hölder solution of $(5)$ on $[0, T]$, with $T>0$, if

$$
\begin{aligned}
& f \in C([0, T], \mathscr{V}) \cap C^{1}\left([0, T], h^{1+\alpha}\left(\mathbb{S}^{1}\right)\right), \\
& v(\cdot, t) \in \operatorname{buc}^{2+\alpha}(G), t \in[0, T],
\end{aligned}
$$

and if $(v, f)$ satisfies the equations (5) pointwise. It is obvious that problems (2) and (5) are equivalent in the following sense: 
Lemma 1.3 Let $f_{0} \in \mathscr{V}$ be given.

(a) If $(u, f)$ is a classical Hölder solution of $(2)$, then $\left(\phi_{f}^{*} u, f\right)$ is a classical Hölder solution of (5).

(b) If $(v, f)$ is a classical Hölder solution of (5), then $\left(\phi_{*}^{f} v, f\right)$ is a classical Hölder solution of (2)

Proof See for example ref. [4].

Lemma 1.4 Given $\vartheta \in(0,1)$ and $k \geqslant 0$, the mapping

$$
C^{k+2+\vartheta}\left(\mathbb{S}^{1}\right) \ni g \longmapsto \kappa_{g}=\frac{g^{\prime \prime}}{\left(1+\left(g^{\prime}\right)^{2}\right)^{3 / 2}} \in C^{k+\vartheta}\left(\mathbb{S}^{1}\right)
$$

is smooth.

Proof The proof follows by direct computation.

Using Lemma 1.3 and general results from the theory of quasilinear elliptic equations we obtain the following existence, uniqueness and regularity result.

Theorem 1.5 Let $f \in \mathscr{V}$ be given. There exists a unique solution $v \in \operatorname{buc}^{2+\alpha}(G)$ of the quasilinear Dirichlet problem

$$
\begin{aligned}
& \mathscr{A}(f) v=0 \quad \text { in } \quad G \text {, } \\
& v=-\gamma \kappa_{f}+f \quad \text { on } \quad \partial G .
\end{aligned}
$$

Denote by $\mathscr{T}(f) \in \operatorname{buc}^{2+\alpha}(G)$ the solution to (6). The mapping $\left[\mathscr{V} \ni f \mapsto \mathscr{T}(f) \in \operatorname{buc}^{2+\alpha}(G)\right]$ is smooth.

\section{The abstract Cauchy problem}

Let $\mathscr{T}: \mathscr{V} \subset h^{4+\alpha}\left(\mathbb{S}^{1}\right) \rightarrow \operatorname{buc}^{2+\alpha}(G)$ be the solution operator defined in Theorem 1.5. We reduce problem (5) into an abstract, nonlinear Cauchy problem on $\mathbb{S}^{1}$

$$
\partial_{t} f+\phi(f)=0, \quad f(0)=f_{0},
$$

where

$$
\phi(f):=\mathscr{B}(f, \mathscr{T}(f)) .
$$

We consider here the restriction $\mathscr{B}: \mathscr{V} \times \operatorname{buc}^{2+\alpha}(G) \rightarrow h^{1+\alpha}\left(\mathbb{S}^{1}\right)$ of the operator defined in Section 1. Given $(f, v) \in \mathscr{V} \times \operatorname{buc}^{2+\alpha}(G)$, we have

$$
\mathscr{B}(f, v)=-\frac{1}{\bar{\mu}\left(\left|\nabla_{f} v\right|^{2}\right)}\left(f^{\prime} \gamma_{0} v_{1}+\frac{1}{f}\left(1+f^{\prime 2}\right) \gamma_{0} v_{2}\right),
$$

where $\nabla_{f} v(x):=D\left(\phi_{*}^{f} v\right)(x, f(x))=\gamma_{0} D_{f} v(x)$ for $x \in \mathbb{S}^{1}$ and $\gamma_{0}$ is the trace operator on $\Gamma_{0}$. The following result on the differentiability of $\mathscr{B}$ holds. 
Lemma 2.1 The mapping $\mathscr{B}: \mathscr{V} \times \operatorname{buc}^{2+\alpha}(G) \rightarrow h^{1+\alpha}\left(\mathbb{S}^{1}\right)$ is smooth. The Fréchet derivative of $\mathscr{B}$ in $(f, v) \in \mathscr{V} \times \operatorname{buc}^{2+\alpha}(G)$ is given by

$$
\begin{aligned}
\partial \mathscr{B}(f, v)[h, u]= & -\frac{1}{\bar{\mu}}\left(\left|\nabla_{f} v\right|^{2}\right)\left[f^{\prime} \gamma_{0} u_{1}+h^{\prime} \gamma_{0} v_{1}+\frac{1}{f}\left(1+f^{\prime 2}\right) \gamma_{0} u_{2}\right. \\
& \left.-\left(\frac{h}{f^{2}}-\frac{2 f^{\prime} h^{\prime}}{f}+\frac{h f^{\prime 2}}{f^{2}}\right) \gamma_{0} v_{2}\right] \\
& -2\left(\frac{1}{\bar{\mu}}\right)^{\prime}\left(\left|\nabla_{f} v\right|^{2}\right)\left(f^{\prime} \gamma_{0} v_{1}+\frac{1}{f}\left(1+f^{\prime 2}\right) \gamma_{0} v_{2}\right)\left[\gamma_{0} v_{1} u_{1}+\frac{h^{\prime}}{f} \gamma_{0} v_{1} v_{2}\right. \\
& +\frac{f^{\prime}}{f} \gamma_{0} u_{1} v_{2}+\frac{f^{\prime}}{f} \gamma_{0} v_{1} u_{2}-\frac{f^{\prime} h}{f^{2}} \gamma_{0} v_{1} v_{2}+\frac{f^{\prime} h^{\prime}}{f^{2}} \gamma_{0} v_{2}^{2}+\frac{f^{\prime 2}}{f^{2}} \gamma_{0} v_{2} u_{2} \\
& \left.-\frac{h f^{\prime 2}}{f^{3}} \gamma_{0} v_{2}^{2}+\frac{1}{f^{2}} \gamma_{0} v_{2} u_{2}-\frac{h}{f^{3}} \gamma_{0} v_{2}^{2}\right],
\end{aligned}
$$

for all $[h, u] \in h^{2+\alpha}\left(\mathbb{S}^{1}\right) \times \operatorname{buc}^{2+\alpha}(G)$.

Lemma 2.2 Let $f \in \mathscr{V}$ and $h \in h^{4+\alpha}\left(\mathbb{S}^{1}\right)$ be given. The function $\partial \mathscr{T}(f)[h]$ is the unique solution of the linear Dirichlet problem

$$
\begin{aligned}
& b_{i j} w_{i j}+b w_{2}+D_{f} w\left[u_{11} \partial a_{11}\left(D_{f} u\right)+2 u_{12}\left(\frac{(1-y) f^{\prime}}{f} \partial a_{11}\left(D_{f} u\right)-\frac{1}{f} \partial a_{12}\left(D_{f} u\right)\right)\right. \\
& +u_{22}\left(\frac{(1-y)^{2} f^{\prime 2}}{f^{2}} \partial a_{11}\left(D_{f} u\right)-2 \frac{(1-y) f^{\prime}}{f^{2}} \partial a_{12}\left(D_{f} u\right)+\frac{1}{f^{2}} \partial a_{22}\left(D_{f} u\right)\right) \\
& \left.+u_{2}\left((1-y)\left(\frac{f^{\prime \prime}}{f}-2 \frac{f^{\prime 2}}{f^{2}}\right) \partial a_{11}\left(D_{f} u\right)+2 \frac{f^{\prime}}{f^{2}} \partial a_{12}\left(D_{f} u\right)\right)\right] \\
& =-u_{2}\left((1-y) \frac{f h^{\prime}-f^{\prime} h}{f^{2}}, \frac{h}{f^{2}}\right) \cdot\left[u_{11} \partial a_{11}\left(D_{f} u\right)\right. \\
& +2 u_{12}\left(\frac{(1-y) f^{\prime}}{f} \partial a_{11}\left(D_{f} u\right)-\frac{1}{f} \partial a_{12}\left(D_{f} u\right)\right)+u_{22}\left(\frac{(1-y)^{2} f^{\prime 2}}{f^{2}} \partial a_{11}\left(D_{f} u\right)\right. \\
& \left.-2 \frac{(1-y) f^{\prime}}{f^{2}} \partial a_{12}\left(D_{f} u\right)+\frac{1}{f^{2}} \partial a_{22}\left(D_{f} u\right)\right)+u_{2}\left((1-y)\left(\frac{f^{\prime \prime}}{f}-2 \frac{f^{\prime 2}}{f^{2}}\right) \partial a_{11}\left(D_{f} u\right)\right. \\
& \left.\left.+2 \frac{f^{\prime}}{f^{2}} \partial a_{12}\left(D_{f} u\right)\right)\right]-2 u_{12}\left((1-y) \frac{f h^{\prime}-f^{\prime} h}{f^{2}} a_{11}\left(D_{f} u\right)+\frac{h}{f^{2}} a_{12}\left(D_{f} u\right)\right) \\
& -2 u_{22}\left(\frac{(1-y)^{2}\left(f f^{\prime} h^{\prime}-f^{\prime 2} h\right)}{f^{3}} a_{11}\left(D_{f} u\right)-(1-y) \frac{f h^{\prime}-2 f^{\prime} h}{f^{3}} a_{12}\left(D_{f} u\right)-\frac{h}{f^{3}} a_{22}\left(D_{f} u\right)\right) \\
& -u_{2}\left((1-y)\left(\frac{f h^{\prime \prime}-f^{\prime \prime} h}{f^{2}}-4 \frac{f f^{\prime} h^{\prime}-f^{\prime 2} h}{f^{3}}\right) a_{11}\left(D_{f} u\right)+2 \frac{f h^{\prime}-2 f^{\prime} h}{f^{3}} a_{12}\left(D_{f} u\right)\right) \text { in } \quad G \text {, } \\
& w=-\gamma \frac{1}{\left(1+\left(f^{\prime}\right)^{2}\right)^{\frac{3}{2}}} h^{\prime \prime}+3 \gamma \frac{f^{\prime} f^{\prime \prime}}{\left(1+\left(f^{\prime}\right)^{2}\right)^{\frac{5}{2}}} h^{\prime}+h \quad \text { on } \quad \partial G,
\end{aligned}
$$


where $u:=\mathscr{T}(f)$ and $b_{i j}=b_{i j}(y, f, D u), b=b(y, f, D u)$ are the coefficients of $\mathscr{A}(f)$. Again $w_{i j}, w_{1}, w_{2}$ denote partial derivatives of $w$.

Proof The proof is standard and is left to the reader.

Combining Lemmas 2.1 and 2.2 we conclude that the nonlocal pseudodifferential operator $\phi$ defined at the very beginning of this section depends smoothly on the function $f$. More precisely, we state:

\section{Theorem 2.3}

$$
\phi \in C^{\infty}\left(\mathscr{V}, h^{1+\alpha}\left(\mathbb{S}^{1}\right)\right)
$$

Given $f \in \mathscr{V}$ we have

$$
\partial \phi(f)=\partial \mathscr{B}(f, \mathscr{T}(f)) \circ\left(i d_{h^{4+\alpha}\left(\mathbb{S}^{1}\right)}, \partial \mathscr{T}(f)\right)
$$

Let $c \in \mathbb{R}_{>0}$ be fixed. Our goal is to prove existence and uniqueness of the solution to (7) for $f_{0}$ in a small neighbourhood $\mathcal{O}$ of $c$ in $h^{4+\alpha}\left(\mathbb{S}^{1}\right)$. To this end we use general results from the theory of maximal regularity, see ref. [12].

We prove next that $-\partial \boldsymbol{\phi}(c)$, regarded as an unbounded operator in $h^{1+\alpha}\left(\mathbb{S}^{1}\right)$, with dense domain $h^{4+\alpha}\left(\mathbb{S}^{1}\right)$ generates a strongly continuous analytic semigroup, i.e. $\partial \phi(c) \in$ $\mathscr{H}\left(h^{4+\alpha}\left(\mathbb{S}^{1}\right), h^{1+\alpha}\left(\mathbb{S}^{1}\right)\right)$.

The solution $\mathscr{T}(c)$ to (6) is the constant function $c$ on $G$. Given $h \in h^{4+\alpha}\left(\mathbb{S}^{1}\right), w:=$ $\partial \mathscr{T}(c)[h]$ is the unique solution in $\operatorname{buc}^{2+\alpha}(G)$ of the Dirichlet problem

$$
\begin{aligned}
c^{2} w_{11}+w_{22} & =0 & & \text { in } \quad G, \\
w & =h-\gamma h^{\prime \prime} & & \text { on } \quad \partial G,
\end{aligned}
$$

and

$$
-\partial \phi(c)[h]=-\partial \mathscr{B}(c, \mathscr{T}(c))[h, w]=\frac{\zeta}{c} \gamma_{0} w_{2},
$$

where $\zeta=1 / \bar{\mu}(0)$.

We consider Fourier expansions of $h$ and $w$

$$
h(x)=\sum_{k \in \mathbb{Z}} c_{k} e^{i k x}, \quad w(x, y)=\sum_{k \in \mathbb{Z}} C_{k}(y) e^{i k x},
$$

and substitute these expressions in (8). Comparing the coefficients of $e^{i k x}, k \in \mathbb{Z}$, we get the following equations for $C_{k}$ :

$$
\begin{aligned}
C_{k}^{\prime \prime}-c^{2} k^{2} C_{k} & =0, \quad 0<y<2, \\
C_{k}(0) & =\left(1+\gamma k^{2}\right) c_{k}, \\
C_{k}(2) & =\left(1+\gamma k^{2}\right) c_{k},
\end{aligned}
$$


for $k \in \mathbb{Z} \backslash\{0\}$, and

$$
\begin{aligned}
C_{0}^{\prime \prime} & =0, \quad 0<y<2, \\
C_{0}(0) & =c_{0} \\
C_{0}(2) & =c_{0} .
\end{aligned}
$$

Using ODE techniques we determine the solution to (10) to be the constant function $C_{0} \equiv c_{0}$, while, for $k \in \mathbb{Z} \backslash\{0\}$, the solution to (9) is

$$
C_{k}(y)=\left(1+\gamma k^{2}\right) c_{k} d_{k}(y), \quad 0 \leqslant y \leqslant 2,
$$

where

$$
d_{k}(y):=\frac{e^{2 c k}-1}{e^{4 c k}-1} e^{c k y}+\frac{e^{4 c k}-e^{2 c k}}{e^{4 c k}-1} e^{-c k y}, \quad 0 \leqslant y \leqslant 2 .
$$

Consequently,

$$
-\partial \phi(c)\left[\sum_{k \in \mathbb{Z}} c_{k} e^{i k x}\right]=\sum_{k \in \mathbb{Z}} \lambda_{k} c_{k} e^{i k x},
$$

for all $h=\sum_{k \in \mathbb{Z}} c_{k} e^{i k x} \in h^{4+\alpha}\left(\mathbb{S}^{1}\right)$, with

$$
\lambda_{k}:=-\zeta \frac{e^{2 c k}-1}{e^{2 c k}+1}\left(k+\gamma k^{3}\right), \quad k \in \mathbb{Z} .
$$

The multiplicative coefficients $\lambda_{k}$ satisfy $\lambda_{k}=\lambda_{-k}$ for all $k \in \mathbb{Z}$ and the sequence $\left(\lambda_{k}\right)_{k \geqslant 0}$ is strictly decreasing to $-\infty$

$$
0=\lambda_{0}>\lambda_{ \pm 1}>\lambda_{ \pm 2}>\cdots>\lambda_{ \pm k}>\lambda_{ \pm(k+1)}>\cdots
$$

We shall make use of this representation of $-\partial \phi(c)$ to prove that $\partial \phi(c)$ belongs to $\mathscr{H}\left(h^{4+\alpha}\left(\mathbb{S}^{1}\right), h^{1+\alpha}\left(\mathbb{S}^{1}\right)\right)$. It is enough, cf. ref. [1], to find constants $\omega>0$ and $\chi \geqslant 1$ such that

$$
\begin{gathered}
\lambda+\partial \phi(c) \in \operatorname{Isom}\left(h^{4+\alpha}\left(\mathbb{S}^{1}\right), h^{1+\alpha}\left(\mathbb{S}^{1}\right)\right), \\
|\lambda| \cdot\|R(\lambda,-\partial \phi(c))\|_{\mathscr{L}\left(h^{1+\alpha}\left(\mathbb{S}^{1}\right)\right)} \leqslant \chi
\end{gathered}
$$

for all $\operatorname{Re} \lambda \geqslant \omega$. Here, given two Banach spaces $\mathbf{E}$ and $\mathbf{F}$, we denote by $\operatorname{Isom}(\mathbf{E}, \mathbf{F})$ the subset of $\mathscr{L}(\mathbf{E}, \mathbf{F})$ containing just isomorphisms, i.e. bijective linear operators. As usually, $\mathscr{L}(\mathbf{E}, \mathbf{F})$ stands for the space of all linear and bounded operators form $\mathbf{E}$ to $\mathbf{F}$. The open map theorem implies especially that the inverse $T^{-1}$ belongs to $\mathscr{L}(\mathbf{F}, \mathbf{E})$, if $T$ is an element of $\operatorname{Isom}(\mathbf{E}, \mathbf{F})$.

For simplicity we choose $\omega=1$. We now study $-\partial \phi(c)$, regarded as an multiplier operator on Sobolev spaces over $\mathbb{S}^{1}$. Given $r \geqslant 0$, the Sobolev space $H^{r}\left(\mathbb{S}^{1}\right)$ is defined by

$$
H^{r}\left(\mathbb{S}^{1}\right):=\left\{f \in L^{2}\left(\mathbb{S}^{1}\right): \sum_{n \in \mathbb{Z}}\left(1+n^{2}\right)^{r}|\widehat{f}(n)|^{2}<\infty\right\},
$$


where $\widehat{f}(n)$ is the $n$th Fourier coefficient of $f$. This space is endowed with the scalar product

$$
\langle f, g\rangle:=\sum_{n \in \mathbb{Z}}\left(1+n^{2}\right)^{r} \widehat{f}(n) \overline{\widehat{g}(n)}
$$

The smooth functions are dense in $H^{r}\left(\mathbb{S}^{1}\right)$ and the Sobolev embedding $H^{k+r}\left(\mathbb{S}^{1}\right) \hookrightarrow C^{k}\left(\mathbb{S}^{1}\right)$ holds for all $k \in \mathbb{N}$, provided $r>1 / 2$. Therefore we have

$$
H^{k+s}\left(\mathbb{S}^{1}\right) \stackrel{d}{\hookrightarrow} h^{k+\beta}\left(\mathbb{S}^{1}\right),
$$

for all $k \in \mathbb{N}, \beta \in[0,1]$ and $s>3 / 2$.

Lemma 2.4 Given $r \geqslant 0$ and $\operatorname{Re} \lambda \geqslant \omega$, we have $\lambda+\partial \phi(c) \in I \operatorname{som}\left(H^{r+3}\left(\mathbb{S}^{1}\right), H^{r}\left(\mathbb{S}^{1}\right)\right)$.

Proof The proof follows directly from $\lim _{k \rightarrow \infty} \lambda_{k} / k^{3}=-\gamma \zeta$. Given $\operatorname{Re} \lambda \geqslant \omega$ and $h=$ $\sum_{k \in \mathbb{Z}} \widehat{h}(k) e^{i k x} \in H^{r+3}\left(\mathbb{S}^{1}\right)$ we have

$$
\begin{aligned}
\left\|\partial \boldsymbol{\phi}(c)\left[\sum_{k \in \mathbb{Z}} \widehat{h}(k) e^{i k x}\right]\right\|_{H^{r}\left(\mathbb{S}^{1}\right)} & =\sum_{k \in \mathbb{Z}}\left(1+k^{2}\right)^{r}\left|\lambda_{k} \widehat{h}(k)\right|^{2} \leqslant\left. M^{2} \sum_{k \in \mathbb{Z}}\left(1+k^{2}\right)^{r+3} \widehat{h}(k)\right|^{2} \\
& =M^{2}\left\|\sum_{k \in \mathbb{Z}} \widehat{h}(k) e^{i k x}\right\|_{H^{r+3}\left(\mathbb{S}^{1}\right)},
\end{aligned}
$$

with $M>0$ such that $\left|\lambda_{k}\right| \leqslant M\left(1+k^{2}\right)^{\frac{3}{2}}, \forall k \in \mathbb{Z}$. Since $\left|\lambda-\lambda_{k}\right| \geqslant 1$ holds for all $k \in \mathbb{Z}$, it follows that $\lambda+\partial \phi(c)$ is injective.

Using the same argument as given earlier it is easy to verify that the linear mapping

$$
H^{r}\left(\mathbb{S}^{1}\right) \ni \sum_{k \in \mathbb{Z}} c_{k} e^{i k x} \longmapsto \sum_{k \in \mathbb{Z}} \frac{1}{\lambda-\lambda_{k}} c_{k} e^{i k x} \in H^{r+3}\left(\mathbb{S}^{1}\right),
$$

is well defined and this completes the proof.

Given $r \geqslant 0$ and $\operatorname{Re} \lambda \geqslant \omega$, the inverse of $\lambda+\partial \phi(c)$ is the operator $R(\lambda,-\partial \phi(c)) \in$ $\operatorname{Isom}\left(H^{r}\left(\mathbb{S}^{1}\right), H^{r+3}\left(\mathbb{S}^{1}\right)\right)$ defined by

$$
R(\lambda,-\partial \boldsymbol{\phi}(c))\left[\sum_{k \in \mathbb{Z}} c_{k} e^{i k x}\right]=\sum_{k \in \mathbb{Z}} \frac{1}{\lambda-\lambda_{k}} c_{k} e^{i k x},
$$

for all $h:=\sum_{k \in \mathbb{Z}} c_{k} e^{i k x} \in H^{r}\left(\mathbb{S}^{1}\right)$.

By virtue of (15) and Lemma 2.4 we obtain

Corollary 2.5 Let $k \in\{1,4\}$ and suppose $R(\lambda,-\partial \phi(c)) \in \mathscr{L}\left(C^{1+\alpha}\left(\mathbb{S}^{1}\right), C^{k+\alpha}\left(\mathbb{S}^{1}\right)\right)$, for some $\operatorname{Re} \lambda \geqslant \omega$. Then $R(\lambda,-\partial \phi(c)) \in \mathscr{L}\left(h^{1+\alpha}\left(\mathbb{S}^{1}\right), h^{k+\alpha}\left(\mathbb{S}^{1}\right)\right)$.

Proof We prove first the case $k=4$. The proof of the assertion in the case $k=1$ is similar. By assumption, we know that $R(\lambda,-\phi(c)) \in \mathscr{L}\left(h^{1+\alpha}\left(\mathbb{S}^{1}\right), C^{4+\alpha}\left(\mathbb{S}^{1}\right)\right)$. Given $f \in h^{1+\alpha}\left(\mathbb{S}^{1}\right)$, 
from (15) we find a sequence $\left(f_{n}\right)_{n} \subset H^{r}\left(\mathbb{S}^{1}\right), r>3$, such that $f_{n} \rightarrow f$ in $C^{1+\alpha}\left(\mathbb{S}^{1}\right)$. Thus

$$
R(\lambda,-\partial \phi(c)) f_{n} \longrightarrow R(\lambda,-\partial \phi(c)) f \quad \text { in } \quad C^{4+\alpha}\left(\mathbb{S}^{1}\right) .
$$

We know that $R(\lambda,-A) f_{n} \in H^{r+3}\left(\mathbb{S}^{1}\right)$. Consequently

$$
R(\lambda,-\partial \phi(c)) f \in{\overline{H^{r+3}\left(\mathbb{S}^{1}\right)}}^{\|\cdot\|_{C^{4+\alpha}\left(\mathbf{S}^{1}\right)}}=h^{4+\alpha}\left(\mathbb{S}^{1}\right) .
$$

Having stated Corollary 2.5 we prove now that the resolvent set $\rho(-\partial \phi(c))$ of the linearization contains an halfplane. More precisely, we have:

\section{Proposition 2.6}

$$
\{\lambda \in \mathbb{C}: \operatorname{Re} \lambda \geqslant \omega\} \subset \rho(-\partial \phi(c)) .
$$

Proof Let $\operatorname{Re} \lambda \geqslant \omega$ be given. In virtue of Corollary 2.5, it is enough to show that $R(\lambda,-\partial \phi(c)) \in \mathscr{L}\left(C^{1+\alpha}\left(\mathbb{S}^{1}\right), C^{4+\alpha}\left(\mathbb{S}^{1}\right)\right)$. Because $R(\lambda,-\partial \phi(c))$ is a multiplier operator (see (16)), we are done if we have

(i) $\sup _{k \in \mathbb{Z}}|k|^{3}\left|M_{k}\right|<\infty$,

(ii) $\sup _{k \in \mathbb{Z}}|k|^{4}\left|M_{k+1}-M_{k}\right|<\infty$, and

(iii) $\sup _{k \in \mathbb{Z}}|k|^{5}\left|M_{k+2}-2 M_{k+1}+M_{k}\right|<\infty$,

where $M_{k}:=1 /\left(\lambda-\lambda_{k}\right)$ (see, e.g., refs. [2] and [13]). From

$$
\lim _{k \rightarrow \infty} \frac{k^{3}}{\lambda-\lambda_{k}}=\frac{1}{\gamma \zeta}
$$

we obtain (i). For $k \geqslant 1$ we have

$$
k^{4}\left|M_{k+1}-M_{k}\right|=\frac{k^{3}}{\left|\lambda-\lambda_{k+1}\right|} \frac{k^{3}}{\left|\lambda-\lambda_{k}\right|} \frac{\left|\lambda_{k+1}-\lambda_{k}\right|}{k^{2}} \underset{k \rightarrow \infty}{\longrightarrow} \frac{3}{\gamma \zeta},
$$

because of $\left(\lambda_{k}-\lambda_{k+1}\right) / k^{2} \rightarrow 3 \gamma \zeta$, (ii) is proved. For $k \geqslant 1$ we compute

$$
\begin{aligned}
k^{5}\left|M_{k+2}-2 M_{k+1}+M_{k}\right|= & \frac{k^{3}}{\left|\lambda-\lambda_{k+2}\right|} \frac{k^{3}}{\left|\lambda-\lambda_{k+1}\right|} \frac{k^{3}}{\left|\lambda-\lambda_{k}\right|} \frac{1}{k^{4}} \mid \lambda\left(\lambda_{k+2}-2 \lambda_{k+1}+\lambda_{k}\right) \\
& +\lambda_{k}\left(\lambda_{k+1}-\lambda_{k+2}\right)+\lambda_{k+2}\left(\lambda_{k+1}-\lambda_{k}\right) \mid,
\end{aligned}
$$

and $\left(\lambda_{k+2}-2 \lambda_{k+1}+\lambda_{k}\right) / k^{4} \rightarrow 0$, respectively $\left(\lambda_{k}\left(\lambda_{k+1}-\lambda_{k+2}\right)+\lambda_{k+2}\left(\lambda_{k+1}-\lambda_{k}\right)\right) / k^{4} \rightarrow 12 \gamma^{2} \zeta^{2}$. This completes the proof.

Proposition 2.7 There exists $\chi \geqslant 1$ such that

$$
|\lambda| \cdot\|R(\lambda,-\partial \phi(c))\|_{\mathscr{L}\left(h^{1+\alpha}\left(\mathbb{S}^{1}\right)\right)} \leqslant \chi
$$

for all $\operatorname{Re} \lambda \geqslant \omega$. 
Proof Given $h=\sum_{k \in \mathbb{Z}} \widehat{h}(k) e^{i k x} \in h^{1+\alpha}\left(\mathbb{S}^{1}\right)$, we have

$$
|\lambda| R(\lambda,-\partial \phi(c))\left[\sum_{k \in \mathbb{Z}} \widehat{h}(k) e^{i k x}\right]=\sum_{k \in \mathbb{Z}} M_{k}^{\lambda} \widehat{h}(k) e^{i k x}
$$

where

$$
M_{k}^{\lambda}=\frac{|\lambda|}{\lambda-\lambda_{k}}, \quad \forall k \in \mathbb{Z}, \operatorname{Re} \lambda \geqslant \omega
$$

It suffices to prove (see refs. [2] and [13]) that there exist positive constants $s_{1}, s_{2}$ and $s_{3}$ such that

(i) $\sup _{k \in \mathbb{Z}}\left|M_{k}^{\lambda}\right| \leqslant s_{1}$,

(ii) $\sup _{k \in \mathbb{Z}}|k|\left|M_{k+1}^{\lambda}-M_{k}^{\lambda}\right| \leqslant s_{2}$, and

(iii) $\sup _{k \in \mathbb{Z}}|k|^{2}\left|M_{k+2}^{\lambda}-2 M_{k+1}^{\lambda}+M_{k}^{\lambda}\right| \leqslant s_{3}$,

for all $\operatorname{Re} \lambda \geqslant \omega$. For $\operatorname{Re} \lambda \geqslant \omega$ and $k \in \mathbb{Z}$ we have $\operatorname{Re}\left(\lambda-\lambda_{k}\right) \geqslant 1$ and $\left|\lambda-\lambda_{k}\right| \geqslant|\lambda|$. Consequently (i) holds with $s_{1}=1$. For $k \neq 0$, we have $\left|\lambda-\lambda_{k}\right| \geqslant\left|\lambda_{k}\right|$, for all $\operatorname{Re} \lambda \geqslant \omega$, hence

$$
|k|\left|M_{k+1}^{\lambda}-M_{k}^{\lambda}\right|=\frac{|\lambda|}{\left|\lambda-\lambda_{k+1}\right|} \frac{|k|^{3}}{\left|\lambda-\lambda_{k}\right|} \frac{\left|\lambda_{k+1}-\lambda_{k}\right|}{k^{2}} \leqslant \frac{|k|^{3}}{\left|\lambda_{k}\right|} \frac{\left|\lambda_{k+1}-\lambda_{k}\right|}{k^{2}},
$$

and (ii) holds. For $k \geqslant 1$ we further have

$$
\begin{aligned}
k^{2}\left|M_{k+2}-2 M_{k+1}+M_{k}\right|= & \frac{|\lambda|}{\left|\lambda-\lambda_{k+2}\right|} \frac{k^{3}}{\left|\lambda-\lambda_{k+1}\right|} \frac{k^{3}}{\left|\lambda-\lambda_{k}\right|} \frac{1}{k^{4}} \mid \lambda\left(\lambda_{k+2}-2 \lambda_{k+1}+\lambda_{k}\right) \\
& +\lambda_{k}\left(\lambda_{k+1}-\lambda_{k+2}\right)+\lambda_{k+2}\left(\lambda_{k+1}-\lambda_{k}\right) \mid \\
\leqslant & \frac{k^{3}}{\left|\lambda_{k}\right|} \frac{\left|\lambda_{k+2}-2 \lambda_{k+1}+\lambda_{k}\right|}{k} \\
& +\frac{k^{3}}{\left|\lambda_{k}\right|} \frac{k^{3}}{\left|\lambda_{k+1}\right|} \frac{\left|\lambda_{k}\left(\lambda_{k+1}-\lambda_{k+2}\right)+\lambda_{k+2}\left(\lambda_{k+1}-\lambda_{k}\right)\right|}{k^{4}} .
\end{aligned}
$$

Since

$$
\frac{\lambda_{k+2}-2 \lambda_{k+1}+\lambda_{k}}{k} \underset{k \rightarrow \infty}{\longrightarrow}-6 \gamma \zeta
$$

we obtain, using the symmetry of the coefficients $\lambda_{k}$, (iii) and the proof is completed.

From (13) and (14) we conclude that $-\partial \phi(c)$ generates a strongly continuous analytic semigroup in $\mathscr{L}\left(h^{1+\alpha}\left(\mathbb{S}^{1}\right)\right)$. The proof of Theorem 1.1 is now similar to that of Theorem 8.1.1 in ref. [12], and the assumptions of this theorem are all satisfied (see Theorem 2.3). 


\section{Stability results}

A pair $(u, f) \in \operatorname{buc}^{2+\alpha}\left(\Omega_{f}\right) \times \mathscr{V}$ is a steady state of the flow (1) iff it is a solution of the free boundary value problem

$$
\begin{aligned}
\operatorname{div}\left(\frac{D u}{\bar{\mu}\left(|D u|^{2}\right)}\right) & =0 & & \text { in } \quad \Omega_{f}, \\
\partial_{v} u & =0 & & \text { on } \quad \Gamma_{0}, \\
u & =-\gamma \kappa_{f}+f & & \text { on } \quad \Gamma_{f}, \\
\partial_{v} u & =0 & & \text { on } \quad \Gamma_{f} .
\end{aligned}
$$

It is obvious that constant functions $(u, f)=(c, c)$, with $c \in \mathbb{R}_{>0}$, are steady-state solutions of system (1). In the following we show that there are in fact no other steady states. Assume that $(u, f)$ is an equilibrium of (1). Then it follows from the first, the second and the last equation of (17) that there exists a constant $c \in \mathbb{R}$ such that $u=c$. Moreover the function $f$ must solve the differential equation

$$
f-c=\gamma \frac{f^{\prime \prime}}{\left(1+\left(f^{\prime}\right)^{2}\right)^{3 / 2}} \quad \text { on } \quad \mathbb{S}^{1} .
$$

Because of the periodicity of $f$ we find a point $x_{0}$ where $f$ attains its maximum $M$. Let us first assume that $M>c$. From $f^{\prime \prime}\left(x_{0}\right)=(M-c) / \gamma>0$ and $f^{\prime}\left(x_{0}\right)=0$ we obtain that $f$ is constant in the neighbourhood of $x_{0}$. The set $f^{-1}(M)$ is therefore a non-empty, closed and open in $\mathbb{S}^{1}$. We conclude that $f=M$, in contradiction with (18).

If $M<c$ we have $f^{\prime \prime}<0$ and we obtain a contradiction to the periodicity of $f$. Consequently $M=c$ and together with (18) we deduce $f=c$.

Summarizing, a pair $(u, f) \in \operatorname{buc}^{2+\alpha}\left(\Omega_{f}\right) \times \mathscr{V}$ is a steady state of the flow iff there exists a positive constant $c$ such that $(u, f)=(c, c)$.

Now we fix $c \in \mathbb{R}_{>0}$, and study the stability of the equilibrium $(c, c) \in \operatorname{buc}^{2+\alpha}\left(\Omega_{c}\right) \times \mathscr{V}$ for the flow (1). The corresponding steady state for the flow (5) is the pair $(c, c) \in$ $\operatorname{buc}^{2+\alpha}(G) \times \mathscr{V}$. Using similar arguments as given in Proposition 2.6 we compute

$$
\left\{\lambda_{k}: k \in \mathbb{N}\right\}=\sigma_{p}(-\partial \phi(c))=\sigma(-\partial \phi(c))
$$

In [6] the spectrum of the linearized operator is discrete and contains only positive eigenvalues. The situation presented here is more complicated since $\lambda_{0}=0$ belongs to the spectrum of the linearization.

We now transfer problem (7) in the neighbourhood of the origin by letting $\mathscr{V}_{c}:=\mathscr{V}-c$ and defining $\psi: \mathscr{V}_{c} \subset h^{4+\alpha}\left(\mathbb{S}^{1}\right) \rightarrow h^{1+\alpha}\left(\mathbb{S}^{1}\right)$ by $\psi(f):=-\boldsymbol{\phi}(f+c)$, for $f \in \mathscr{V}_{c}$. We have to study now the stability of the equilibrium $f=0$ for the abstract Cauchy problem

$$
\partial_{t} f=\psi(f), \quad f(0)=f_{0}
$$

Let $\widetilde{C}^{\infty}\left(\mathbb{S}^{1}\right):=\left\{f \in C^{\infty}\left(\mathbb{S}^{1}\right): \int_{\mathbb{S}^{1}} f d x=0\right\}$. For $k \in \mathbb{N}$, let $\widetilde{h}^{k+\alpha}\left(\mathbb{S}^{1}\right)$ be the closure of $\widetilde{C}^{\infty}\left(\mathbb{S}^{1}\right)$ in $C^{k+\alpha}\left(\mathbb{S}^{1}\right)$. In this way we have defined closed subspaces of the little Hölder 
spaces. We have $\widetilde{h}^{4+\alpha}\left(\mathbb{S}^{1}\right) \stackrel{d}{\hookrightarrow} \widetilde{h}^{1+\alpha}\left(\mathbb{S}^{1}\right)$ and

$$
\widetilde{h}^{k+\alpha}\left(\mathbb{S}^{1}\right)=\left\{f \in h^{k+\alpha}\left(\mathbb{S}^{1}\right): \int_{\mathbb{S}^{1}} f d x=0\right\},
$$

for all $k \in \mathbb{N}$. Let $\widetilde{\mathscr{V}}:=\mathscr{V}_{c} \cap h^{4+\alpha}\left(\mathbb{S}^{1}\right)$.

Lemma 3.1 (Conservation of volume) Given $g \in \widetilde{\mathscr{V}}$, we have $\psi(g) \in \widetilde{h}^{1+\alpha}\left(\mathbb{S}^{1}\right)$.

Proof Let $g+c=f \in \mathscr{V}$ and denote by $u$ the solution of (4). We have

$$
\begin{aligned}
\int_{\mathbb{S}^{1}} \psi(g) d x & =-\int_{\mathbb{S}^{1}} \phi(f) d x=-\int_{\mathbb{S}^{1}} \mathscr{B}(f, \mathscr{T}(f)) d x \\
& =-\int_{\mathbb{S}^{1}} \frac{D u}{\bar{\mu}\left(|D u|^{2}\right)}(x, f(x)) \cdot n(x) d x=-\frac{1}{2 \pi} \int_{\Gamma_{f}} \frac{\partial_{v} u}{\bar{\mu}\left(|D u|^{2}\right)} d \sigma \\
& =-\frac{1}{2 \pi} \int_{\Omega_{f}} \operatorname{div}\left(\frac{D u}{\bar{\mu}\left(|D u|^{2}\right)}\right) d x+\frac{1}{2 \pi} \int_{\Gamma_{0}} \frac{\partial_{v} u}{\bar{\mu}\left(|D u|^{2}\right)} d \sigma=0 .
\end{aligned}
$$

This motivates us to consider the restriction $\widetilde{\psi}$ of $\psi$ to $\widetilde{\mathscr{V}}$. From Theorem 2.3 we have that $\widetilde{\psi}$ belongs to $C^{\infty}\left(\widetilde{\mathscr{V}}, \widetilde{h}^{1+\alpha}\left(\mathbb{S}^{1}\right)\right)$ and

$$
\partial \widetilde{\psi}(0)\left[\sum_{k \in \mathbb{Z} \backslash\{0\}} \widehat{h}(k) e^{i k x}\right]=\sum_{k \in \mathbb{Z} \backslash\{0\}} \lambda_{k} \widehat{h}(k) e^{i k x},
$$

for all $h=\sum_{k \in \mathbb{Z} \backslash\{0\}} \widehat{h}(k) e^{i k x} \in \widetilde{h}^{4+\alpha}\left(\mathbb{S}^{1}\right)$. The coefficients $\left\{\lambda_{k}\right\}_{k \in \mathbb{Z} \backslash\{0\}}$ are given by (12). Because the functions $h \in h^{4+\alpha}\left(\mathbb{S}^{1}\right)$ have integral mean equal to zero, that is $\widehat{h}(0)=0$, we can invert $\partial \widetilde{\psi}(0)$.

Even more, we have the following result:

Theorem 3.2 Consider $\partial \widetilde{\psi}(0)$ as an unbounded operator in $\widetilde{h}^{1+\alpha}\left(\mathbb{S}^{1}\right)$ with dense domain $\widetilde{h}^{4+\alpha}\left(\mathbb{S}^{1}\right)$. Then

$$
\begin{gathered}
\sigma(\partial \widetilde{\psi}(0))=\left\{\lambda_{k}: k \geqslant 1\right\}, \\
|\lambda| \cdot\|R(\lambda,-\partial \widetilde{\psi}(0))\|_{\mathscr{L}\left(\widetilde{h}^{1+\alpha}\left(\mathbf{S}^{1}\right)\right)} \leqslant \chi, \quad \forall \operatorname{Re} \lambda \geqslant \omega,
\end{gathered}
$$

where $\chi$ and $\omega$ are the constants from Proposition 2.7 .

Summarizing, - $-\partial \widetilde{\psi}(0)$ belongs to $\mathscr{H}\left(\widetilde{h}^{4+\alpha}\left(\mathbb{S}^{1}\right), \widetilde{h}^{1+\alpha}\left(\mathbb{S}^{1}\right)\right)$ and therefore, if the initial value $f_{0}$ in (19) belongs to $\widetilde{h}^{4+\alpha}\left(\mathbb{S}^{1}\right)$ and is small enough, then this problem possesses a unique solution and the evolution takes place entirely in $\widetilde{h}^{4+\alpha}\left(\mathbb{S}^{1}\right)$.

Combining Theorem 3.2 and Theorem 9.1.2 of ref. [12] we obtain the stability result stated in Theorem 1.2. 


\section{Conclusion}

Starting from a generalized version of Darcy's law we have derived a mathematical model for the dynamic of a Stokesian fluid situated between the parallel plates of a vertical Hele-Shaw cell. Comparing it to ref. [4], the inclusion of surface tension effects is the new important feature of this work. The Newtonian case $\mu \equiv$ constant, treated in refs. [6-9], is included in this model.

The corresponding moving boundary problem is a coupled system consisting of a quasilinear mixed boundary problem for the velocity potential $u$ and a fully nonlinear evolution equation for the interface $f$. We transformed the problem into an abstract Cauchy problem for $f$ on the unit circle $\mathbb{S}^{1}$ :

$$
\partial_{t} f+\phi(f)=0, \quad f(0)=f_{0}
$$

and attacked it by investigating the Fréchet derivative of $\boldsymbol{\phi}$. Using the theory of maximal regularity given by Lunardi [12], we proved the existence and uniqueness of local solutions for initial data $f_{0}$ sufficiently close to a positive constant. The volume of the fluid is preserved by the flow.

In fact, the constant functions are the only equilibria of this system. Different equilibria correspond to different quantities of fluid. Prescribing a volume of fluid, there exists a unique steady state with the property that the domain determined by it contains exactly this volume of fluid. This steady state attracts all solutions at an exponential rate, provided they are initially close enough to the cylinder determined by the steady state and enclose the same volume of fluid.

\section{Acknowledgement}

We thank the editors and the anonymous reviewers for very helpful remarks and suggestions.

\section{References}

[1] Amann, H. (1995) Linear and Quasilinear Parabolic Problems, Vol. I, Birkhäuser, Basel, Switzerland.

[2] Arendt, W. \& BU, S. (2004) Operator-valued Fourier multipliers on periodic Besov spaces and applications, Proceedings of the Edinburgh Mathematical Society 47, 15-33.

[3] Escher, J. (1997) On moving boundaries in deformable media. Adv. Math. Sci. Appl. 7(1), 275-316.

[4] Escher, J. \& Matioc, B.-V. (2008) A moving boundary problem for periodic Stokesian Hele-Shaw flows, Interfaces and Free Boundaries, to appear.

[5] Escher, J. \& Matioc, B.-V. (2008) Stability of the equilibria for periodic Stokesian Hele-Shaw flows. J. Evol. Eq., online 8.7.2008.

[6] Escher, J. \& Prokert, G. (2001) Stability of the equilibria for spatially periodic flows in porous media. Nonlin. Anal. 45, 1061-1080.

[7] Escher, J. \& Simonett, G. (1991) Maximal regularity for a free boundary problem. Nonlin. Diff. Eq. Appl. 2, 463-510.

[8] Escher, J. \& Simonett, G. (1996) Analyticity of the interface in a free boundary problem. Math. Ann. 305, 435-459. 
[9] Escher, J. \& Simonett, G. (1997) Classical solutions of multidimensional Hele-Shaw models. SIAM J. Math. Anal. 28(5), 1028-1047.

[10] Gilbarg, D. \& Trudinger, T. S. (1997) Elliptic Partial Differential Equations of Second Order, Springer-Verlag, New York.

[11] Kondic, L., Palffy-Mahorny, P. \& Shelley, M. J. (1996) Models of non-Newtonian HeleShaw flow. Phys. Rev. E 54(5), R4536-R4539.

[12] Lunardi, A. (1995) Analytic Semigroups and Optimal Regularity in Parabolic Problems, Birkhäuser, Basel, Switzerland.

[13] Schmeisser, H.-J. \& Triebel H. (1987) Topics in Fourier Analysis and Function Spaces, John Wiley and Sons, New York.

[14] Shaughnessy, E. J., Katz, I. M. \& Schaffer, J. P. (2005) Introduction to Fluid Mechanics, Oxford University Press, New York. 